\title{
Efeitos da Terapia Espelho na funcionalidade do membro superior pós- AVC: revisão integrativa
}

\author{
Effects of Mirror Therapy on upper limb functionality post- \\ stroke: integrative review
}

\section{Efectos de la Terapia con Espejos en la funcionalidad de la miembro superior post-ACV: revisión integrativa}

\author{
Elisson de Sousa Mesquita Silva ${ }^{1}$, Ana Carolina Silva Barros ${ }^{1}$, \\ Antonia Mairla Nascimento de Brito ${ }^{1}$, Igor Oliveira Lima Rodrigues ${ }^{1}$, \\ Hyorranne Raysa Lima Maximiano ${ }^{1}$, Elivelton Sousa Montelo², \\ Jessica Inara Brito de Siqueira², Lucas da Silva Nascimento², \\ Tassiane Maria Alves Pereira ${ }^{3}$, Victor Hugo do Vale Bastos ${ }^{4}$
}

1.Graduando(a) em Fisioterapia pela Universidade Federal do Delta do Parnaíba, Parnaíba-PI, Brasil. 2.Fisioterapeuta pela Universidade Federal do Piauí, Parnaíba-PI, Brasil.

3.Fisioterapeuta, Especialista em Fisioterapia Hospitalar pela Faculdade Inspirar, Teresina-PI, Brasil. 4.Fisioterapeuta, Doutor em Psiquiatria e Saúde Mental pela Universidade Federal do Rio de Janeiro, Docente da Universidade Federal do Delta do Parnaíba, Parnaíba-PI, Brasil.

\begin{abstract}
Resumo
Introdução. O Acidente Vascular Cerebral (AVC) é um acometimento neurológico que pode ser classificado em dois tipos: isquêmico e hemorrágico, capazes de causarem uma série de alterações motoras, cognitivas e sensoriais. Nesse contexto, a Terapia Espelho é um recurso de baixo custo, implementado no tratamento para membros paralisados. Objetivo. Reunir informações através de uma busca na literatura a fim de verificar os efeitos da Terapia espelho na funcionalidade do membro superior afetado de pacientes pós-acidente vascular cerebral. Método. Foi realizada uma revisão integrativa da literatura por meio da busca de estudos nas bases de dados PubMed, BVS; CINAHL; Embase; Web of Science; Scopus. A seleção dos artigos se deu em quatro etapas: levantamento bibliográfico utilizando as palavras-chaves "Stroke" AND "paresis" OR "upper extremity" AND "mirror therapy", leitura do título, leitura do resumo e leitura na íntegra dos artigos. Resultados. Foram identificados 310 estudos, dos quais 13 foram avaliados pela escala PEDro, tornando-se elegíveis para a revisão, de acordo com os critérios estabelecidos. Conclusão. A Terapia Espelho associada ou não a outras intervenções foi capaz de melhorar a funcionalidade do membro superior afetado de indivíduos, nos aspectos motores, sensitivos, atividade de vida diária, e proporcionou qualidade de vida dos pacientes pós-AVC.
\end{abstract}

Unitermos. Acidente vascular cerebral; Paresia; Membro Superior; Reabilitação

\footnotetext{
Abstract

Introduction. Stroke is a neurological disorder that can be classified into two types: ischemic and hemorrhagic, capable of causing a series of motor, cognitive and sensory changes. In this context, mirror therapy is a low-cost resource, implemented in the treatment of paralyzed limbs. Objective. Gather information through a search in the literature to verify the effects of mirror therapy on the functionality of the affected upper limb of post-stroke patients. Method. An integrative literature review was conducted by searching the PubMed, BVS; CINAHL; Embase; Web of Science; and Scopus databases. The selection of articles took place in four stages: bibliographic search using the keywords "Stroke" AND "paresis" OR "upper extremity" AND "mirror therapy", reading of the title, reading of the abstract and reading of the full articles. Results. 310 studies were identified, of which 13 were evaluated by the PEDro scale, making them eligible for review according to the established criteria. Conclusion. Mirror Therapy associated or not with other interventions was able to improve the functionality of the
} 
affected upper limb of individuals, in motor and sensory aspects, activities of daily living, and provided quality of life for post-stroke patients.

Keywords. Stroke; Paresis; Upper extremity; Rehabilitation

\begin{abstract}
Resumen
Introducción. El accidente cerebrovascular ( $A C V)$ es un trastorno neurológico que se puede clasificar en dos tipos: isquémico y hemorrágico, capaz de provocar una serie de alteraciones motoras, cognitivas y sensoriales. En este contexto, la terapia del espejo es un recurso de bajo costo, implementado en el tratamiento de miembros paralizados. Objetivo. recopilar información a través de una búsqueda bibliográfica para verificar los efectos de la terapia del espejo sobre la funcionalidad del miembro superior afectado de pacientes post-accidente cerebrovascular. Método. Se realizó una revisión integradora de la literatura mediante la búsqueda de estudios en PubMed, BVS; CINAHL; Embase; Web de la Ciencia; Scopus. La selección de los artículos se realizó en cuatro etapas: levantamiento bibliográfico utilizando las palabras clave "accidente cerebrovascular" $Y$ "paresia" O "extremidad superior" $Y$ "terapia del espejo", lectura de títulos, lectura de resúmenes y lectura completa de los artículos. Resultados. Se identificaron un total de 310 estudios, de los cuales 13 fueron evaluados por la escala PEDro, siendo elegibles para revisión, según los criterios establecidos. Conclusión. La Terapia del Espejo, asociada o no a otras intervenciones, logró mejorar la funcionalidad de la extremidad superior afectada de los individuos, en términos de aspectos motores y sensoriales, actividades de la vida diaria y proporcionó calidad de vida a los pacientes postaccidente cerebrovascular.
\end{abstract}

Palabras clave. Accidente cerebrovascular; Paresia; Miembro superior; Rehabilitación

Trabalho realizado na Universidade Federal do Delta do Parnaíba, Parnaíba-PI, Brasil.

Conflito de interesse: não

Recebido em: 22/10/2021

Aceito em: 06/12/2021

Endereço de correspondência: Victor Hugo do Vale Bastos. Universidade Federal do Piauí. Av. São Sebastião 2819. Nossa Sra. de Fátima. Parnaíba-PI, Brasil. CEP 64202-020. Email: victorhugobastos@ufpi.edu.br

\title{
INTRODUÇÃO
}

O Acidente Vascular cerebral (AVC) refere-se a uma síndrome neurológica aguda, que pode acontecer mediante uma obstrução dos vasos sanguíneos, levando ao AVC isquêmico, ou pode acontecer uma ruptura desses vasos, levando ao AVC hemorrágico, sendo o primeiro mais comum na população ${ }^{1}$.

Conforme a Organização Mundial da Saúde (OMS), o AVC é considerado a segunda causa de morte no mundo, causando cerca de 6,7 milhões de óbitos. A tendência é mantida até o ano de 2030 , respondendo por $12,2 \%$ do total estimado de mortos ${ }^{2}$. No Brasil, 99.732 mortes foram causadas por doenças cerebrovasculares no ano de 2010 . 0 
laudo médico de AVC, em 2012, foi responsável por aproximadamente 166 mil hospitalizações, a um custo de mais de R\$163 milhões de reais; logo, é considerada uma doença altamente custosa ${ }^{3}$.

Os prejuízos causados pelo AVC podem ser variados, tendo características motoras, cognitivas e sensoriais. A doença pode acarretar uma redução da força do membro superior (MMSS) e/ou inferior, perda súbita da visão, disfunções na fala, intensa dor de cabeça, desequilíbrio, distúrbios comportamentais, sensibilidade e deglutição, além de outras evidências clínicas comuns, como a hemiplegia e hemiparesia ${ }^{4}$. Essas complicações podem repercutir consideravelmente de modo negativo na independência em atividades laborais, provocando restrições em atividades de vida diária 5 .

As implicações pós-AVC variam de acordo com a localização da lesão e a extensão de seu envolvimento. $O$ mais comum é a função motora prejudicada, que pode incluir disfunções nos membros superiores, inferiores e do tronco ${ }^{6}$.

A função está diretamente relacionada ao desempenho físico, que são as atividades do indivíduo durante o dia e as atividades que ele pode realizar. Portanto, avaliar a independência funcional dessas pessoas após um AVC é essencial para a análise das atividades de vida diária, para que se possam propor reais intervenções de tratamento da condição da vítima e aprimorar o atendimento para determinar as necessidades individuais de cada pessoa?. 
$\mathrm{Na}$ fisioterapia, um recurso alternativo para membros paralisados é a terapia espelho (TE), uma ferramenta simples, de baixo custo, fácil compreensão e implementação. Baseia-se na estimulação visual, no qual os pacientes pósAVC movem seu membro não afetado enquanto observam seu reflexo no espelho sobreposto ao membro afetado (invisível), objetivando ativar os circuitos neurais a reorganizar o córtex e melhorar a função motora ${ }^{8,9}$.

Desse modo, destaca-se a necessidade da compreensão dos déficits motores causados pelo AVC, bem como, a utilização de ferramentas terapêuticas que visam contribuir para um melhor direcionamento para a prática clínica dos profissionais envolvidos com os programas de reabilitação. Portanto, objetivou-se reunir informações através de uma busca na literatura a fim de verificar os efeitos da Terapia espelho na funcionalidade do membro superior afetado de pacientes pós-Acidente Vascular cerebral.

\section{MÉTODO}

Este estudo trata-se de uma Revisão Integrativa (RI) que buscou reunir e apresentar artigos relevantes através da análise crítica acerca da temática dos efeitos da terapia espelho na função do membro superior de pacientes pósacidente vascular cerebral, além de proporcionar direcionamentos para a prática clínica e futuras pesquisas ${ }^{10}$. Os métodos para a condução de RI apresentam algumas etapas a serem seguidas: identificação do tema, seleção da hipótese ou questão norteadora da pesquisa; definição dos 
critérios de inclusão e exclusão; identificação e seleção dos estudos; avaliação dos artigos incluídos; interpretação e exposição dos resultados dos estudos da inclusão final ${ }^{11}$.

De acordo com a primeira etapa, esta pesquisa buscou identificar os efeitos da terapia espelho na funcionalidade do membro superior pós-acidente vascular cerebral, baseandose na questão norteadora: "Quais os efeitos da terapia espelho na funcionalidade do membro superior de pacientes pós-acidente vascular cerebral?".

Desse modo, a busca foi realizada no decorrer do mês de maio de 2021 nas bases de dados PubMed; Biblioteca Virtual em Saúde (BVS); Embase; CINAHL; Scopus; Web of Science; utilizando os descritores da saúde (DeCS) e Medical Subject Headings (MeSH) em associação com os operadores booleanos "AND" e "OR" nas buscas avançadas de títulos e resumos, os descritores foram: stroke AND paresis OR upper extremity AND mirror therapy.

Incluiu-se estudos de ensaio clínico randomizados originais e estudos pilotos randomizados, em qualquer idioma, disponíveis na integra, publicados entre os anos de 2016 e 2021, que apresentaram escore $\geq 6$ na escala PEDro, estes deveriam abordar a utilização da terapia espelho com ou sem associação a outras terapias na recuperação da funcionalidade do membro superior de pacientes pósacidente vascular cerebral.

Como critérios de exclusão foi estabelecido artigos de pesquisa bibliográfica; teses, dissertações de mestrado, carta ao editor, estudos incompletos, artigos duplicados em 
diferentes bases de dados e aqueles que não respondiam à questão norteadora da pesquisa.

Os artigos foram exportados no formato "BibTeX" para uma ferramenta online de Revisão sistemática da literatura "Parsif.al" com o propósito de exclusão dos artigos duplicados de forma automatizada. Após isso, os estudos foram selecionados mediante a leitura dos títulos e resumos, logo em seguida os que foram selecionados foram analisados através da leitura do texto completo com a finalidade de confirmar os critérios de elegibilidade estabelecidos.

A qualidade metodológica dos artigos foi avaliada conforme os critérios da escala PEDro ${ }^{12}$. Esta escala é composta por 11 itens, sabendo que o primeiro não é contabilizado, obtém-se uma pontuação de 1 a $10^{13,14}$.

As informações relevantes dos trabalhos selecionadas foram extraídas e sumarizadas em um quadro apresentado na seguinte ordem: autor, ano de publicação, amostra, intervenção, variáveis analisadas, desfecho primário e secundário e resultados.

\section{RESULTADOS}

Foram encontrados 310 artigos por meio das bases de dados pesquisadas, 105 foram excluídos por estarem duplicados, 142 por título; 43 por resumo; 7 por texto completo. Ademais, 13 estudos foram incluídos para uma avaliação criteriosa pela escala PEDro, todos contemplavam os critérios de inclusão (Figura 1). 
Figura 1. Fluxograma de seleção dos estudos.

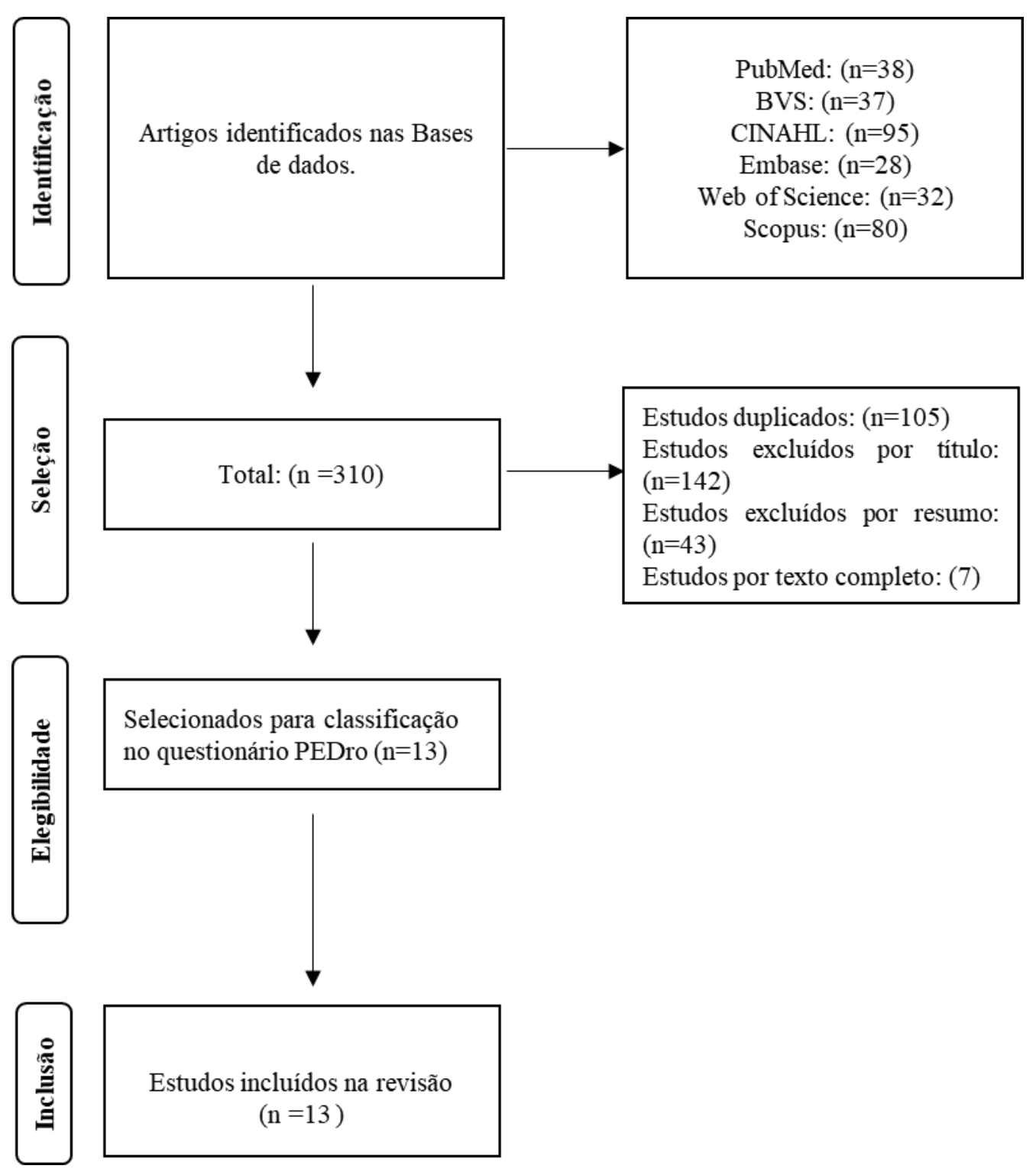

Os artigos analisados contemplaram um total de 478 pacientes com diagnóstico clínico de Acidente Vascular cerebral, avaliando a recuperação motora do membro superior (Escala Fugl-Meyer Assessment-FMA), sensorial (Nottingham Sensory Assessment-NSA), atividade de vida diária (Functional Independence Measure-FIM; Modified Barthel index-MBI), qualidade de vida (Short-Form8-SF8) (Quadro 1). 
Quadro 1. Características dos estudos incluídos, expondo o tamanho da amostra, aplicação da intervenção, desfechos e resultados atingidos.

\begin{tabular}{|c|c|c|c|c|c|}
\hline 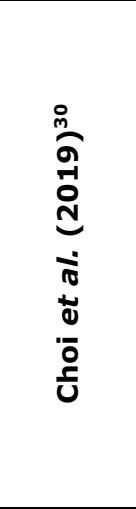 & $\begin{array}{l}36 \quad \text { pacientes } \\
\text { com AVC } \\
\text { crônico. } \\
\text { GR }(n=12) . \quad \\
\text { Terapia de } \\
\text { espelho } \\
\text { convencional } \\
(n=12) . \\
\text { Grupo controle } \\
(n=12) .\end{array}$ & $\begin{array}{l}\text { GR: realizou movimento 3D } \\
\text { terapia espelho baseado em } \\
\text { um dispositivo. } \\
\text { Terapia de espelho } \\
\text { convencional: terapia de } \\
\text { espelho geral. } \\
\text { Grupo controle: Terapia } \\
\text { simulada. } \\
\text { Os grupos passaram por um } \\
\text { programa de treinamento que } \\
\text { resultou em } 15 \text { sessões, } 30 \\
\text { min/dia, } 3 \text { dias por semana, } \\
\text { durante } 5 \text { semanas. Após } 5 \\
\text { semanas, as avaliações finais } \\
\text { foram realizadas. }\end{array}$ & $\begin{array}{l}\text { DP: função } \\
\text { motora. } \\
\text { DS: desconforto } \\
\text { no pescoço, } \\
\text { qualidade de } \\
\text { vida. }\end{array}$ & $\begin{array}{l}\text { MTF: movimento } \\
\text { do } \quad \text { membro } \\
\text { superior, preensão } \\
\text { e manipulação de } \\
\text { resina. } \\
\text { NDS: medir grau } \\
\text { de desconforto } \\
\text { subjetivo no } \\
\text { pescoço. } \\
\text { SF-8: avaliar } \\
\text { satisfação com a } \\
\text { vida. }\end{array}$ & $\begin{array}{l}\text { Melhora significante na } \\
\text { função da extremidade } \\
\text { superior, depressão e } \\
\text { qualidade de vida no grupo } \\
\text { de terapia de espelho GR, } \\
\text { mais do que no grupo de } \\
\text { controle. As mudanças de } \\
\text { desconforto no pescoço na } \\
\text { terapia e controle do } \\
\text { espelho convencional, os } \\
\text { grupos foram } \\
\text { significantemente maiores } \\
\text { do que no grupo de terapia } \\
\text { de espelho GR. }\end{array}$ \\
\hline
\end{tabular}


Quadro 1 (cont.). Características dos estudos incluídos, expondo o tamanho da amostra, aplicação da intervenção, desfechos e resultados atingidos.

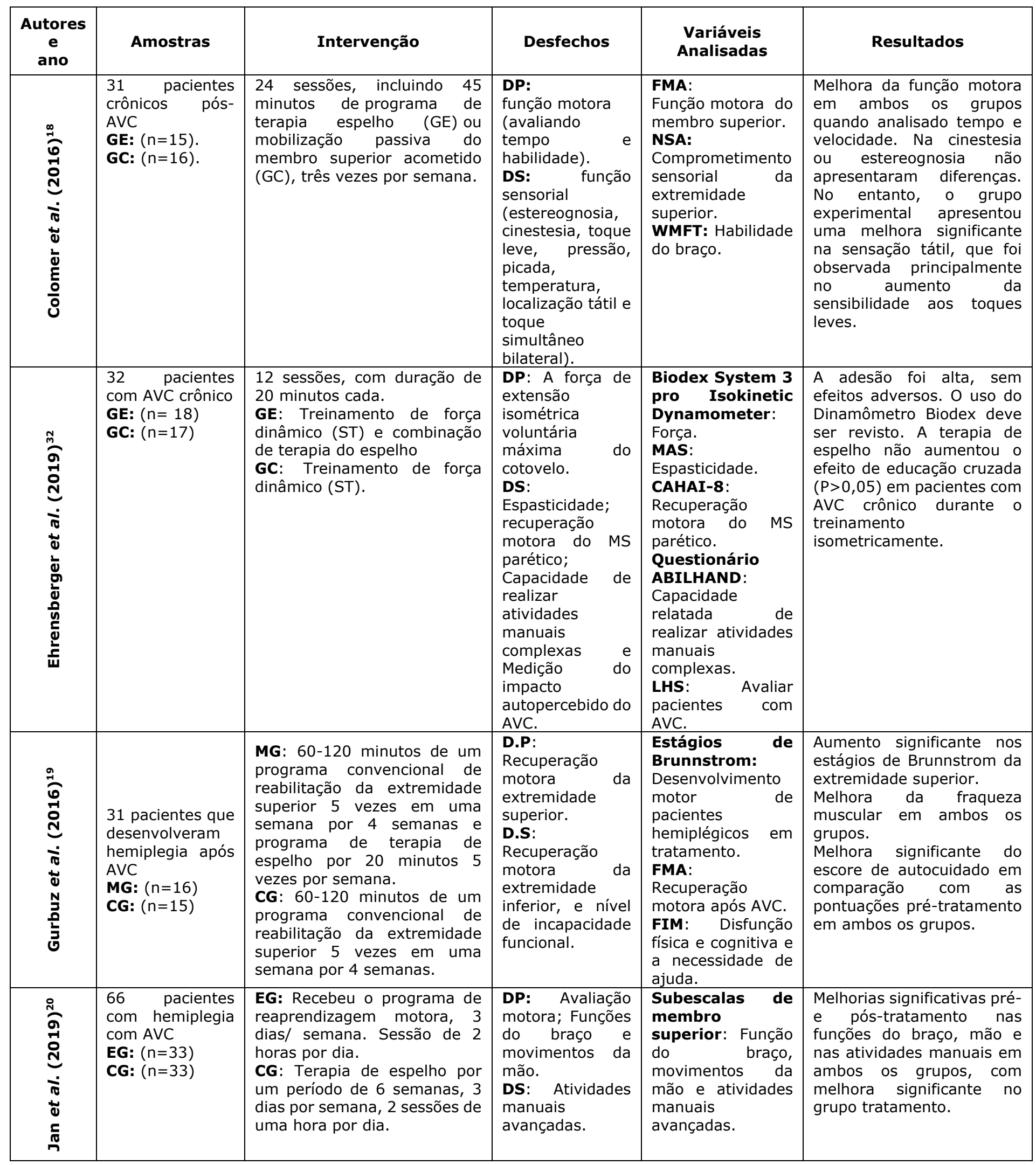


Quadro 1 (cont.). Características dos estudos incluídos, expondo o tamanho da amostra, aplicação da intervenção, desfechos e resultados atingidos.

\begin{tabular}{|c|c|c|c|c|c|}
\hline $\begin{array}{c}\text { Autores } \\
\text { e } \\
\text { ano }\end{array}$ & Amostras & Intervenção & Desfechos & $\begin{array}{l}\text { Variáveis } \\
\text { Analisadas }\end{array}$ & Resultados \\
\hline 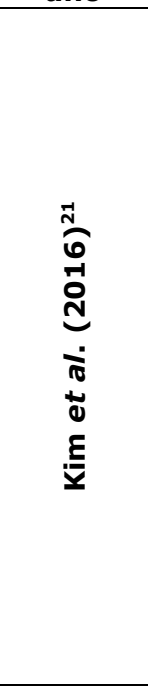 & $\begin{array}{l}25 \text { pacientes } \\
\text { com AVC. } \\
\text { MT*: }(n=12) \\
\text { CT: }(n=13)\end{array}$ & $\begin{array}{l}\text { Sessão de exercícios de } 30 \\
\text { minutos por dia, cinco dias por } \\
\text { semana, em um total de } \\
\text { quatro semanas. } \\
\text { MT*: realizaram atividades } \\
\text { com espelho. } \\
\text { CT: sessões de treinamento } \\
\text { para a melhoria da função dos } \\
\text { membros superiores e } \\
\text { atividades de vida diária. }\end{array}$ & $\begin{array}{lr}\text { DP: Função dos } \\
\text { membros } \\
\text { superiores e nas } \\
\text { atividades da } \\
\text { vida diária. }\end{array}$ & $\begin{array}{lr}\text { ARAT: } & \text { Identificar } \\
\text { a } & \text { melhoria } \\
\text { funcional r da } & \text { capacidade de } \\
\text { desempenho de } \\
\text { um } r \text { membro } \\
\text { superior. } \\
\text { BBT: Mede o } \\
\text { número de peças } \\
\text { de madeira } \\
\text { passadas em um } \\
\text { minuto de uma } \\
\text { mão para a outra. } \\
\text { FMA: Função } \\
\text { motora no ombro, } \\
\text { punho, antebraço, } \\
\text { mão e dedos pós- } \\
\text { AVC. } \\
\text { FIM: Atividades } \\
\text { de vida diária. }\end{array}$ & $\begin{array}{l}\text { A terapia do espelho é mais } \\
\text { eficaz do que a terapia } \\
\text { convencional para o } \\
\text { treinamento de pacientes } \\
\text { com AVC para melhorar a } \\
\text { função dos membros } \\
\text { superiores e as atividades } \\
\text { da vida diária. Melhora } \\
\text { significante no grupo } \\
\text { terapia de espelho em } \\
\text { comparação com o grupo } \\
\text { de terapia convencional, } \\
\text { tanto na função dos } \\
\text { membros superiores } \\
\text { quanto nas atividades da } \\
\text { vida diária. }\end{array}$ \\
\hline 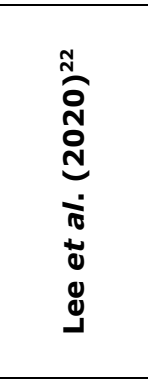 & $\begin{array}{l}21 \quad \text { pacientes } \\
\text { com AVC } \\
\text { EG: }(n=11) \\
\text { CG: }(n=10)\end{array}$ & $\begin{array}{l}\text { Terapia realizada por } 4 \\
\text { semanas, cinco vezes por } \\
\text { semana por } 30 \text { min por dia } \\
\text { EG: realizou tarefas } \\
\text { complexas usando terapia de } \\
\text { espelho baseada em múltiplas } \\
\text { articulações para membro } \\
\text { superior afetado. } \\
\text { CG: realizou terapia de } \\
\text { espelho com foco em uma } \\
\text { única articulação. }\end{array}$ & $\begin{array}{l}\text { DP: Função da } \\
\text { extremidade } \\
\text { superior. } \\
\text { DS: atividade de } \\
\text { vida diária. }\end{array}$ & $\begin{array}{l}\text { FMA: } r \text { Função } \\
\text { motora, braço, } \\
\text { punho e mão. } \\
\text { MAL: Qualidade } \\
\text { do movimento e } \\
\text { quantidade de uso } \\
\text { do braço afetado. } \\
\text { K-MBI: atividade } \\
\text { de vida diária. }\end{array}$ & $\begin{array}{l}\text { GE apresentou melhora em } \\
\text { FMA; MAL e } \quad \text { K-MBI } \\
\text { respectivamente } \\
\text { comparado ao GC. }\end{array}$ \\
\hline 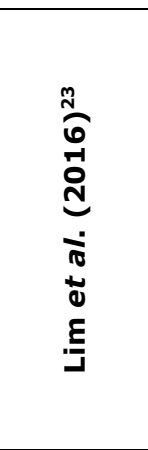 & $\begin{array}{l}60 \quad \text { pacientes } \\
\text { com hemiplegia } \\
\text { pós AVC } \\
\text { EG: }(n=30) . \\
\text { CG: }(n=30) .\end{array}$ & $\begin{array}{l}\text { EG: realizaram tarefas por } 20 \\
\text { minutos, imitando o reflexo do } \\
\text { membro superior normal } \\
\text { como no espelho, } 20 \text { vezes } \\
\text { por } 3 \text { séries com intervalo de } \\
2 \text { minutos entre as séries. } \\
\text { CG: Executou as mesmas } \\
\text { tarefas funcionais no mesmo } \\
\text { período. Uma placa de } \\
\text { madeira foi colocada entre o } \\
\text { membro hemiplégico e o } \\
\text { membro normal em vez de um } \\
\text { espelho }\end{array}$ & $\begin{array}{ll}\text { DP: Melhora } & \text { da } \\
\text { função } & \text { do } \\
\text { membro } & \\
\text { superior. } & \\
\text { DS: Melhora } & \text { na } \\
\text { capacidade } & \text { de } \\
\text { realizar } & \\
\text { atividades } & \text { da } \\
\text { vida diária. } & \end{array}$ & $\begin{array}{l}\text { FMA e } \\
\text { Avaliaram a } \\
\text { função motora do } \\
\text { membro } \\
\text { hemiplégico. } \\
\text { MBI: Avaliou } \\
\text { atividade de vida } \\
\text { diária. }\end{array}$ & $\begin{array}{l}\text { Melhora significante em } \\
\text { ambos os grupos, na função } \\
\text { da extremidade superior do } \\
\text { lado afetado e na } \\
\text { capacidade de realizar } \\
\text { atividades de vida diária, } \\
\text { bem como no FMA e MBI } \\
\text { foram maiores no grupo } \\
\text { terapia espelho. }\end{array}$ \\
\hline 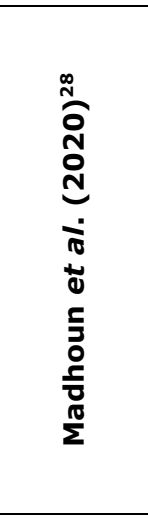 & $\begin{array}{l}30 \text { participantes } \\
\text { com AVC e } \\
\text { hemiparesia dos } \\
\text { membros } \\
\text { superiores. } \\
\text { TBMT: }(n=15) . \\
\text { CG: }(n=15) .\end{array}$ & $\begin{array}{l}25 \text { sessões de tratamento. } \\
\text { TBMT: realizou } 25 \text { minutos de } \\
\text { tarefa funcional com espelho } \\
\text { todos os dias além de terapia } \\
\text { convencional, se necessária, } \\
\text { como terapia manual e } \\
\text { acupuntura. } \\
\text { CG: Terapia ocupacional sem } \\
\text { espelho por } 15 \text { minutos, além } \\
\text { da terapia convencional, se } \\
\text { necessário. }\end{array}$ & $\begin{array}{l}\text { DP: Melhora nas } \\
\text { atividades de } \\
\text { vida diária, } \\
\text { recuperação } \\
\text { motora e função } \\
\text { motora. }\end{array}$ & $\begin{array}{l}\text { FMA: Função } \\
\text { motora no ombro, } \\
\text { punho, antebraço, } \\
\text { mão e dedos pós- } \\
\text { AVC. } \\
\text { BRS: } \\
\text { Desempenho de } \\
\text { recuperação } \\
\text { motora do } \\
\text { membro superior } \\
\text { (braço e mão). } \\
\text { MBI: Atividade de } \\
\text { vida diária. } \\
\text { MAS: } \\
\text { Espasticidade }\end{array}$ & $\begin{array}{l}\text { Não foram observadas } \\
\text { diferenças significantes } \\
\text { entre os grupos no BRS e } \\
\text { no BMI. } \\
\text { Melhora significante no } \\
\text { grupo } \\
\text { TBMT do que no grupo } \\
\text { controle, FMA e certos } \\
\text { aspectos da MAS. }\end{array}$ \\
\hline
\end{tabular}


Quadro 1 (cont.). Características dos estudos incluídos, expondo o tamanho da amostra, aplicação da intervenção, desfechos e resultados atingidos.

\begin{tabular}{|c|c|c|c|c|c|}
\hline $\begin{array}{c}\text { Autores } \\
\text { e } \\
\text { ano }\end{array}$ & Amostras & Intervenção & Desfechos & $\begin{array}{l}\text { Variáveis } \\
\text { Analisadas }\end{array}$ & Resultados \\
\hline 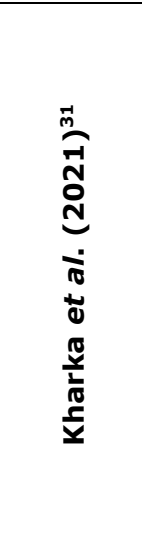 & $\begin{array}{l}40 \quad \text { pacientes } \\
\text { com AVC. } \\
\text { MT: }(n=20) \\
\text { NMES: }(n=20)\end{array}$ & $\begin{array}{l}\text { Os dois grupos receberam os } \\
\text { mesmos programas de } \\
\text { reabilitação convencionais e, } \\
\text { adicionalmente, tiveram cada } \\
\text { uma de suas próprias terapias } \\
\text { por } 30 \text { minutos, } 5 \text { dias por } \\
\text { semana por três semanas. O } \\
\text { programa convencional foi } \\
\text { específico do paciente e } \\
\text { consistiu em desenvolvimento } \\
\text { neurológico, técnicas de } \\
\text { facilitação, fisioterapia, } \\
\text { terapia ocupacional e terapia } \\
\text { da fala (se necessário). }\end{array}$ & $\begin{array}{l}\text { DP: } \\
\text { Recuperação } \\
\text { motora, função } \\
\text { motora } \\
\text { DS: qualidade de } \\
\text { vida. }\end{array}$ & $\begin{array}{l}\text { ARAT e MAS: } \\
\text { medir a melhoria } \\
\text { na recuperação } \\
\text { motora ra do } \\
\text { membro superior. } \\
\text { FMA e MBI: } \\
\text { avaliar } \\
\text { funcionamento } \\
\text { motor. } \\
\text { A qualidade de } \\
\text { vida relacionada à } \\
\text { saúde (HQROL) } \\
\text { foi avaliada pela } \\
\text { qualidade de vida } \\
\text { específica do AVC } \\
\text { (SSQOL). }\end{array}$ & $\begin{array}{l}\text { Melhora significante após } 3 \\
\text { semanas em termos de } \\
\text { recuperação motora, } \\
\text { funcionamento motor e } \\
\text { qualidade de vida. Nenhum } \\
\text { efeito sobre a espasticidade } \\
\text { foi obtido em ambos os } \\
\text { grupos. }\end{array}$ \\
\hline
\end{tabular}

EG= Experimental group; GC= Control Group; TBMT= Task-based mirror therapy; CT**= Control Therapy; MT= Mirror Therapy; MG= Mirror Group; $\mathbf{C G}=$ Convencional Group; $\mathbf{D P}=$ Desfecho Primário; DS = Desfecho Secundário; FMA= Fugl-Meyer Assessment; $\mathbf{B R S}=$ Brunnstrom Assessment; $\mathbf{M B I}=$ Modified Barthel index; MAS= Modified Ashworth Scale; MAL= Motor Activity log; K-MBI= Korean version of the Modified Barthel Index; GR= Gesture recognation; $\mathbf{M T} *=$ mirror therapy combined with exercise; CT= conventional therapy; $\mathbf{A R A T}=$ action research arm test; $\mathbf{B B T}=$ Box and Block Test; $\mathbf{F I M}=$ Functional Independence Measure; NMES= neuromuscular electrical stimulation; HQROL= Health-related quality of life; $\mathbf{S S - Q O L = ~ s t r o k e - s p e c i f i c ~}$ quality of life; $\mathbf{M T F}=$ Manual funcion test; WMFT-time = Wolf Motor Function Test-Time; WMFT= Wolf Motor Function Test; WMTF-FAS=Wolf Motor Function Test-Functional Ability Scale; MMSE = Mini-Mental State Examination; NSA= Nottingham Sensory Assessment; NDS= Neck discomfort score; SF-8= Short- Form 8; , CT*= conventional treatment; MMT= Movement-based mirror therapy; TMT= Task-based mirror therapy; FMA-UE= FuglMeyer Assessment-upper extremity; MAS=Modified Ashworth scale; CAHAI-8= The Chedoke Arm and Hand Activity Inventory Version 8; LHS= The London Handicap Scale; MT: Mirror therapy. SWM= Semmes Weinstein Monofilament 2PD= 2-Point Discrimination.

Em relação a avaliação dos estudos incluídos pela escala PEDro, todos apresentaram boa qualidade metodológica, sendo que, 8 alcançaram escore total 10, e 5 obtiveram score 8 (Quadro 2).

\section{DISCUSSÃO}

O trabalho em questão evidenciou os efeitos da terapia espelho na funcionalidade do membro superior em pacientes pós-AVC. Os estudos verificaram que os grupos de tratamento com a terapia espelho associada a exercícios funcionais, obtiveram melhora significante na função motora do braço acometido ${ }^{15-23}$. 
Quadro 2. Avaliação dos estudos pela escala PEDro.

\begin{tabular}{|l|c|c|c|c|c|c|c|c|c|c|c|}
\hline $\begin{array}{l}\text { PEDro Itens } \\
\text { Autores }\end{array}$ & $\mathbf{2}$ & $\mathbf{3}$ & $\mathbf{4}$ & $\mathbf{5}$ & $\mathbf{6}$ & $\mathbf{7}$ & $\mathbf{8}$ & $\mathbf{9}$ & $\mathbf{1 0}$ & $\mathbf{1 1}$ & $\begin{array}{c}\text { Score } \\
\text { Total }\end{array}$ \\
\hline Madhoun et al. $^{28}$ & 1 & 1 & 1 & 1 & 1 & 1 & 1 & 1 & 1 & 1 & $10 / 10$ \\
\hline Lim et al. $^{23}$ & 1 & 1 & 1 & 1 & 1 & 1 & 1 & 1 & 1 & 1 & $10 / 10$ \\
\hline Lee et al. $^{22}$ & 1 & 1 & 1 & 1 & 0 & 0 & 1 & 1 & 1 & 1 & $8 / 10$ \\
\hline Kim et al. ${ }^{21}$ & 1 & 1 & 1 & 1 & 1 & 1 & 1 & 1 & 1 & 1 & $10 / 10$ \\
\hline Kharka et al. $^{31}$ & 1 & 1 & 1 & 1 & 1 & 1 & 1 & 1 & 1 & 1 & $10 / 10$ \\
\hline Jan et al. ${ }^{20}$ & 1 & 1 & 1 & 1 & 0 & 0 & 1 & 1 & 1 & 1 & $8 / 10$ \\
\hline Gurbuz et al. ${ }^{19}$ & 1 & 1 & 1 & 1 & 1 & 1 & 1 & 1 & 1 & 1 & $10 / 10$ \\
\hline Ehrensberger et al. ${ }^{32}$ & 1 & 1 & 1 & 1 & 1 & 1 & 0 & 0 & 1 & 1 & $8 / 10$ \\
\hline Colomer et al. ${ }^{18}$ & 1 & 1 & 1 & 1 & 1 & 1 & 0 & 0 & 1 & 1 & $8 / 10$ \\
\hline Choi et al. ${ }^{30}$ & 1 & 1 & 1 & 1 & 1 & 1 & 1 & 1 & 1 & 1 & $10 / 10$ \\
\hline Chan et al. ${ }^{17}$ & 1 & 1 & 1 & 1 & 1 & 1 & 0 & 0 & 1 & 1 & $8 / 10$ \\
\hline Bai et al. ${ }^{16}$ & 1 & 1 & 1 & 1 & 1 & 1 & 1 & 1 & 1 & 1 & $10 / 10$ \\
\hline Arya et al. ${ }^{15}$ & 1 & 1 & 1 & 1 & 1 & 1 & 1 & 1 & 1 & 1 & $10 / 10$ \\
\hline
\end{tabular}

itens: 2- alocação aleatória; 3- alocação oculta; 4- comparabilidade da linha de base; 5-7 cegamento (sujeito, terapeuta ou avaliador); 8- acompanhamento adequado; 9- intenção de tratamento; 10- comparações entre grupos; 11- medidas de precisão e variabilidade. Cada critério da escala, é atribuído (1) ponto à presença do indicador de qualidade das evidências e zero (0) à ausência desses indicadores, sabendo que o primeiro item não é contabilizado, obtém-se um somatório de 10 pontos.

Está bem documentado na literatura que uma das teorias da terapia espelho envolve a estimulação cerebral por meio de informações visuais aferentes do membro sadio sobrepostas ao membro paralisado, estas informações visuais excitam áreas motoras primárias do cérebro relacionadas à um membro, que se comunicam com áreas cerebrais do membro oposto, essa ilusão visual pode aumentar a excitabilidade de áreas corticais muscular, capaz de restaurar a função motora por meio da reorganização de outras áreas do cérebro que substitui as áreas danificadas pelo $A V C^{24}$. Esta informação pode explicar os achados do presente estudo, relacionados à melhora significativa que os 
pacientes obtiveram na função motora do membro afetado após a intervenção.

Os estudos de Arya et al. ${ }^{15}$ e Colomer et al. ${ }^{18}$ também analisaram outras variáveis, sendo elas o comprometimento sensorial da extremidade superior, bem como a diminuição da sensação cutânea tátil da palma da mão respectivamente, onde verificou-se melhora significativa de $30 \%$ na resposta ao toque e limiar cutâneo bem como na sensação tátil aos toques leves após o tratamento. Esse aspecto do comprometimento sensorial pode atingir cerca de $70 \%$ dos pacientes com limitações motoras do membro superior, muitas vezes não é muito destacado, mas está estritamente ligado ao nível de recuperação motora ${ }^{25}$.

Relacionado a isso, o sistema somatossensorial inclui todos os elementos do sistema nervoso central e periférico relacionados à transmissão de mensagens somatossensoriais. Os estímulos são enviados até o tálamo projetando-se para neurônios de terceira geração, mediante ligações, do talo-cortical ao córtex parietal, finalizando no córtex somatossensorial primário e secundário e em áreas centrais do telencéfalo, como a ínsula e os gânglios da base ${ }^{26}$.

A terapia espelho pode ser considerada uma ferramenta promissora para a reabilitação do AVC ao que se refere a melhora do comprometimento sensorial, haja visto que no estudo de Kwon et al. ${ }^{27}$ ao examinar os efeitos da integração multissensorial visuo-tátil no córtex sensório motor primário esquerdo em indivíduos saudáveis, descobriram que essa 
estimulação induziu ativações corticais somatossensoriais estendidas em ambos os hemisférios do córtex sensório motor primário esquerdo, córtex somatossensorial secundário bem como no hemisfério direito do córtex parietal posterior ao compararem com a estimulação apenas tátil, induzindo assim uma reorganização cortical sensório-motora durante a realização de tarefas.

Os achados do presente artigo mostraram que os pacientes submetidos a TE obtiveram êxito nos aspectos da atividade de vida diária (AVD), quando avaliados pela ferramenta do índice de Barthel modificado (MBI) $22,23,28$. Este mesmo aspecto também foi avaliado por Yang et al. ${ }^{29} \mathrm{em}$ sua revisão sistemática com metanálise, ao investigar os efeitos da TE na função motora, na AVD e na percepção da dor em sobreviventes de AVC. O desfecho geral mostrou que a TE pode de fato melhorar a função motora e a AVD assim como o alívio da dor. Além disso, a recuperação funcional motora do membro afetado pode proporcionar diminuição da dependência às suas atividades.

Tendo em vista essas melhorias, pode-se perceber que a qualidade de vida diária também pode ser beneficiada, como foi mostrado nos trabalhos de Choi et al. ${ }^{30}$ e Kharka et al. ${ }^{31}$, ambos utilizaram a TE associados a outros métodos terapêuticos como a realidade virtual e a estimulação elétrica neuromuscular, respectivamente. Essa melhora se deu porque os indivíduos sentiram-se motivados mediante a participação voluntária durante o processo de tratamento. Essa adesão também foi alcançada no estudo de 
Ehrensberger et al. ${ }^{32}$, entretanto, a literatura é escassa ao avaliarem o aspecto da qualidade de vida na TE, mostrando a necessidade de novos estudos de intervenção que foquem a qualidade de vida como desfecho primário em MMSS.

\section{CONCLUSÃO}

Diante das evidências abordadas, observou-se que a Terapia espelho associada ou não a outras intervenções é capaz de proporcionar melhorias na função motora do membro afetado em indivíduos acometidos pelo AVC, bem como na sensibilidade cutânea, nas atividades de vida diária, consequentemente, evolução do desempenho funcional e da qualidade de vida destes pacientes.

\section{AGRADECIMENTOS}

O presente trabalho foi realizado com apoio da Coordenação de Aperfeiçoamento de Pessoal de Nível Superior, Brasil (CAPES), Código de Financiamento 001.

\section{REFERÊNCIAS}

1.Almeida PMVD, Bazan R, Pontes-Neto O, Minelli C, Corrente JE, Modolo GP, et al. Translation, cross-cultural adaptation and validation of the Cincinnati prehospital stroke scale in Brazil. Arq Neuropsiquiatr 2021;79:272-7. https://doi.org/10.1590/0004-282X-anp-2020-0246

2.Araújo JPD, Darcis JVV, Tomas ACV, Mello WAD. Tendência da mortalidade por acidente vascular cerebral no Município de Maringá, Paraná entre os anos de 2005 a 2015. Int J Cardiovasc Sci 2018;31:5662. https://doi.org/10.5935/2359-4802.20170097

3.Santos LB, Waters C. Perfil epidemiológico dos pacientes acometidos por acidente vascular cerebral: revisão integrativa. Braz J Develop 2020;6:2749-75. https://doi.org/10.34117/bjdv6n1-198

4. Medeiros PK, Costa AP, Alves ESRC, Souza HMGA. Brain vascular accident : associated factors and impacts on the life of young adults. J Med Health Prom 2021;6:12-22.

https://jmhp.unifip.edu.br/index.php/jmhp/article/view/47/25 
5. Eichinger FLF, Soares AV, Novelleto F, Júnior YS, Filho PB, Domenech $\mathrm{SC}$. Serious game for locomotor rehabilitation of hemiparetic stroke patients. Fisioter Mov 2020;33:15-25.

https://dx.doi.org/10.1590/1980-5918.033.A016

6.Yoshida HM, Barreira J, Fernandes PT. Habilidade motora, sintomas depressivos e função cognitiva em pacientes pós-AVC. Fisioter Pesq 2016;26:9-14. https://doi.org/10.1590/1809-2950/17001026012019 7.Vieira IP, Roca KF, Benites JE, Oliveira JHM, Pereira TM, Lescano FA, et al. Funcionalidade e qualidade de vida em pacientes pós acidente vascular cerebral. Braz J Develop 2020;6:17391-403. https://doi.org/ 10.34117/bjdv6n4-056

8.Saha S, Sur M, Chaudhuri GR, Agarwal S. Effects of mirror therapy on oedema, pain and functional activities in patients with poststroke shoulder-hand syndrome: A randomized controlled trial. Physiother Res Int 2021;26:1-8. https://doi.org/10.1002/pri.1902

9.Silva AA, Vieira KS. A eficácia da terapia espelho no processo de recuperação motora e funcional em pacientes com acidente vascular encefálico. Rev Aten Saúde 2017;15:103-9.

https://doi.org/10.13037/ras.vol15n53.4699

10.Roxa GN, Alorim ARV, Caldas GRF, Rodrigues FEA, Gonçalves MOSS, et al. Perfil Epidemiológico Dos Pacientes Acometidos Com Avc Isquêmico Submetidos a Terapia Trombolítica: Uma Revisão Integrativa / Epidemiological Profile of Patients Affected With Ischemic Stroke Subject To Thrombolytic Therapy: an Integrative Review. Braz J Develop 2021;7:7341-51. https://doi.org/10.34117/bjdv7n1-496

11.Machado VMS, Coimbra AK, Trindade PAS, Campos PIC, Ferreira PC, Brasil RR. Atuação do fisioterapeuta nos cuidados paliativos em pacientes adultos: revisão integrativa. Rev Eletr Acervo Saúde 2021;13:1-11. https://doi.org/10.25248/REAS.e6493.2021

12.Shiwa SR, Costa LOP, Moser ADL, Aguiar IC, Oliveira LVF. PEDro: a base de dados de evidências em fisioterapia. Fisioter Mov 2011;24:523-33. https://doi.org/10.1590/S0103-

51502011000300017

13. Carvalho AR, Souza IM, Silva DH, Silva AR, Adad RBSF, Silva VO. Os efeitos do exercício físico em pacientes submetidos à hemodiálise: uma revisão sistemática. Rev Pesqui Fisioter 2020;10:309-16. https://doi.org/10.17267/2238-2704rpf.v10i2.2638

14.Pereira TMA, Nubes NSM, Nascimento JSF, Nascimento JKF, Azizi MAA, Moreno AM, et al. A vibração focal na espasticidade de pacientes com patologias neurológicas: revisão. Fisioter Bras 2021;21:619-24. https://doi.org/10.33233/fb.v21i6.4546

15.Arya KN, Pandian S, Vikas, Puri V. Mirror Illusion for Sensori-Motor Training in Stroke: A Randomized Controlled Trial. J Stroke Cerebrovasc Dis 2018;27:3236-46.

https://doi.org/10.1016/j.jstrokecerebrovasdis.2018.07.012

16.Bai Z, Zhang J, Zhang Z, Su T, Niu W. Comparison Between Movement-Based and Task-Based Mirror Therapies on Improving Upper Limb Functions in Patients With Stroke: A Pilot Randomized Controlled Trial. Front Neurol 2019;10:288. 
https://doi.org/10.3389/fneur.2019.00288

17.Chan WC, Au-Yeung SSY. Recovery in the Severely Impaired Arm Post-Stroke after Mirror Therapy: A Randomized Controlled Study. Am

J Phys Med Rehabil 2018;97:572-7. https://doi.org/10.1097/ PHM.0000000000000919

18.Colomer C, Noé E, Llorens R. Mirror therapy in chronic stroke survivors with severely impaired upper limb function: A randomized controlled trial. Eur J Phys Rehabil Med 2016;52:271-8. https://www.minervamedica.it/en/journals/europamedicophysica/article.php?cod=R33Y2016N03A0271

19.Gurbuz N, Afkar SI, Ayaş S, Cosar SNS. Effect of mirror therapy on upper extremity motor function in stroke patients: a randomized controlled trial. J Phys Ther Sci 2016;28:2501-6.

https://doi.org/10.1589/jpts.28.2501

20.Jan S, Arsh A, Darain H, Gul S. A randomized control trial comparing the effects of motor relearning programme and mirror therapy for improving upper limb motor functions in stroke patients. J Pak Med Assoc 2019;69:1242-5.

http://www.jcreview.com/fulltext/33-1531306084.pdf

21.Kim K, Lee S, Kim D, Lee K, Kim Y. Effects of mirror therapy combined with motor tasks on upper extremity function and activities daily living of stroke patients. J Phys Ther Sci 2016;28:483-7. https://doi.org/10.1589/jpts.28.483

22. Lee SH, Park JS, Choi JB, Yoo W. Improving upper extremity motor function in stroke patients using a complex task with multi-joint-based mirror therapy: A randomized controlled trial. Neurol Asia 2020;25:245-51. http://www.neurology-asia.org/articles/neuroasia2020-25(3)-245.pdf

23.Lim KB, Lee HJ, Yoo J, Yun J. Efficacy of mirror therapy containing functional tasks in poststroke patients. Ann Rehabil Med 2016;40:62936. https://doi.org/10.5535/arm.2016.40.4.629

24. Thieme $\mathrm{H}$, Morkisch N, Mehrholz J, Pohl M, Behrens J, Borgetto B, et al. Mirror therapy for improving motor function after stroke. Cochrane Database Syst Rev 2018;7:CD008449. https://doi.org/ 10.1002/14651858.CD008449.pub3

25. Meyer S, De Bruyn N, Lafosse C, Van Dijk M, Michielsen M, Thijs L, et al. Somatosensory Impairments in the Upper Limb Poststroke. Neurorehabil Neural Repair 2016;30:731-42. https://doi.org/10.1177/ 1545968315624779

26.Kessner SS, Bingel U, Thomalla G. Somatosensory deficits after stroke: a scoping review. Top Stroke Rehabil 2016;23:136-46. https://doi.org/10.1080/10749357.2015.1116822

27.Kwon HG, Jang SH, Lee MY. Effects of visual information regarding tactile stimulation on the somatosensory cortical activation: a functional MRI study. Neural Regen Res 2017;12:1119. https://doi.org/10.4103/1673-5374.211191

28. Madhoun HY, Tan B, Feng Y, Zhou Y, Zhou C, Yu L. Task-based mirror therapy enhances the upper limb motor function in subacute stroke patients: a randomized control trial. Eur J Phys Rehabil Med 
2020;56:265-71. https://doi.org/10.23736/S1973-9087.20.06070-0 29.Yang Y, Zhao Q, Zhang Y, Wu Q, Jiang X, Cheng G. Effect of Mirror Therapy on Recovery of Stroke Survivors: A Systematic Review and Network Meta-analysis. Neuroscience 2018;390:318-36. https://doi.org/10.1016/j.neuroscience.2018.06.044

30.Choi HS, Shin WS, Bang DH. Mirror therapy using gesture recognition for upper limb function, neck discomfort, and quality of life after chronic stroke: A single-blind randomized controlled trial. Med Sci Monit 2019;25:3271-8. https://doi.org/10.12659/MSM.914095

31. Kharka M, Singh P. A Study to Compare the Effectiveness of Mirror Therapy and Neuromuscular Electrical Stimulation on Upper-Extremity Motor Recovery, Motor Function, and Quality of Life in Subacute Stroke Subjects: A Randomized Controlled Trial. Med J DY Patil Vidyapeeth 2021; 14:318-26.

https://doi.org/10.4103/mjdrdypu.mjdrdypu 191_19

32. Ehrensberger M, Simpson D, Broderick P, Blake C, Horgan F, Hickey $P$, et al. Unilateral Strength Training and Mirror Therapy in Patients with Chronic Stroke: A Pilot Randomized Trial. Am J Phys Med Rehabil 2019;98:657-65. https://doi.org/10.1097/phm.0000000000001162 